\title{
Classroom-based Conversation Analytic Research: Theoretical and Applied Perspectives on Pedagogy
}

Silvia Kunitz, Numa Markee, and Olcay Sert (Eds.). 2021. Springer. Pp. v + 426.

At the heart of Kunitz, Markee, and Sert's (2021) edited volume is the question of how classroom interaction can contribute to second language acquisition. Using conversation analysis (CA) as a methodological tool to answer this question, the editors and contributing authors of this book also endeavor to package their findings in ways that are accessible for all kinds of language teaching professionals (e.g., pre- and in-service teachers, language program administrators, teacher supervisors, etc.). Their main argument for doing so hinges on the fact that research can sometimes occur in a vacuum, and that stakeholders need to develop a "consumers' understanding" (p. 1) of how to translate findings from CA in order to inform curricular change.

The book is thematically organized into six parts. It starts with two preliminary chapters that situate the rest of the chapters. First, the editors introduce the motivation behind the major goals of the book and some of the theoretical underpinnings behind CA and ethnomethodology. Notably, they explain that each chapter of the book ends with a final section of pedagogical implications in an attempt to develop stronger systematic links between theory and application. This is informed by their own understanding of curricular innovation as predicated on an active exchange of ideas between all stakeholders in a language learning environment. Next, Pekarek Doehler shows how current thinking about language development has shifted from a focus on individualistic and cognitive approaches to a conception that language learning emerges in and through social interaction. To this end, she unpacks the term, interactional competence (IC), which refers to one's ability to navigate social interaction (through, for example, turn-taking, opening or closing a conversation, disagreeing, etc.) across diverse interactional contexts. Language learning is thus conceived of in terms of the development of interactional competence which entails the diversification of members' methods for accomplishing social action. This chapter ends by summarizing how this conception of interactional competence can inform pedagogy in terms of activity development, teacher training, and designing assessments.

The next section of the book is devoted to CA research conducted in a variety of classroom contexts (e.g., L2 Swedish, L2 English, and L2 Italian classrooms). Together, these studies provide an in-depth understanding of classroom interactional practices, and, in some cases, their longitudinal development, in order to illuminate how these practices contribute to (or are perhaps even indicative of) second language learning. First, Majilesi shows how objects of learning, or "learnables," (e.g., an unknown vocabulary word) emerge as a result of participants' own interaction with each other and their physical environment. This is clearly demonstrated in Majilesi's first example where the topicalization of "rumpa" ("backside" in English) transitions from being a "laughable," where a student tries to use it jokingly, into a learnable as the student realizes that they do not know the word and require assistance (which other students and the teacher ultimately provide). In the next chapter, Eskildsen shows how a beginning L2 speaker of English gradually develops the interactional resources necessary to accomplish a routinized classroom activity (writing the dates of today, yesterday, and tomorrow on the board).

(C) 2022 Romig. This is an open access article distributed under the terms of the Creative Commons Attribution License, which permits the user to copy, distribute, and transmit the work provided that the original authors and source are credited. 
Specifically, he shows how one student's repertoire of embodied, interactional, and linguistic resources expanded so that they could participate in the activity more fully by volunteering, writing, accounting for their own responses, and even eliciting volunteers for subsequent activities. After that chapter, Musk takes a closer look at how spelling corrections are accomplished in a computer-assisted collaborative writing task in an L2 English classroom. Essentially, he finds that these sequences of spelling correction are guided by the typist's epistemic access to the correction. In other words, if the typist knows the correct spelling, they will have the first opportunity to notice a misspelling and give the correction; otherwise, either the teacher and/or computer will be consulted to collaboratively fix the misspelled word. Lastly, Kunitz' analysis of instruction-giving sequences in an L2 Italian classroom shows that the sequence becomes progressively minimized as students become progressively more familiar with the task at hand. In particular, Kunitz shows how the first instruction-giving sequence is full of repair and additional information about what to do, whereas subsequent instruction-giving sequences are minimal, only including a short summons-answers sequence.

The third section of the book narrows the focus by exclusively showcasing CA research in content-based language classrooms. First, Evnitskaya explores how collaborative learning unfolds in two different classroom subject contexts. Essentially, she shows how students' own orientations to managing facework (i.e., their ability to monitor and respond to possibly facethreatening acts) are evident in their use of specific linguistic and multimodal resources. For example, students completing a task in a mathematics classroom displayed a strong preference for affiliation and agreement through the use of L1s and positive assessments of group members' contributions. Pivoting to physics and history classrooms, Kääntä shows how teachers' definitional practices utilize a variety of linguistic and multimodal resources (e.g., drawings already established on the board) to provide definitions that are context-sensitive and recipientdesigned for students. Although the definitional practices of the teachers differed in various ways, particularly with respect to the kind of subject-specific language used, they were united in that they were contingent upon students' problems in understanding. Lee concludes this section by exploring teachers' ordering decisions (i.e., shifting from one topic to the next) in ESL and EFL classrooms. Building upon the widely established Initiation-Reply-Feedback sequence, Lee shows how teachers will rearrange topics during their lesson to respond to students' problems in understanding. A clear example of this is when a teacher delayed their explanation of a concept in order to address students' lack of understanding of a related vocabulary item.

The fourth section focuses on the use of CA research to inform teacher education, particularly through the exploration of different frameworks. First, Sert analyzes three different extracts of code switching by two different teachers to establish comparative ways of how teachers can manage knowledge check questions. He argues that these analyses, and their corresponding audio-visual recordings can be usefully integrated into his IMDAT teacher education framework which involves: (1) (I)ntroduction to classroom interactional competence, (2) (M)icro-teaching experience, (3) (D)ialogic reflection of video-recorded teaching practice with a mentor, (4) (A)nother round of teaching, and (5) (T)eacher collaboration for peer feedback. Sert ends by exploring the use of the VEO App (http://www.veo-group.com), a practical tool for gathering audio-visual material that can be tagged in a way that can correlate well with CA-based findings (e.g., the use of gesture or materials). Second, Waring centers the notion of heteroglossia (i.e., the multifunctional role of the same spate of teacher talk) in the development of her teacher education framework, SWEAR. She proposes that cultivating novice teachers' awareness of their heteroglossic talk can be accomplished by (1) (S)ituating a problem, 
(2) (W)orking with a recording or transcript of classroom interaction, (3) (E)xpanding discussions with other examples, (4) (A)rticulating strategies that can be helpful in other contexts, and (5) (R)ecording and reflecting one's own teaching. Whereas Waring and Sert dealt with the use of CA on classroom recordings to inform their frameworks, Kim and Silver conclude this section by using CA to examine how mentors and novice teachers navigate postobservation conversations. Their analysis shows that mentors come to enact two different kinds of roles, feedback-provider vs. facilitator of reflection, depending on their use of various linguistic and multimodal resources. For example, a characteristic feature of feedback-oriented episodes involved the mentor initiating with a "what do you think about X" question whereas reflection-oriented episodes included much more prefatory work (e.g., establishing mutual gaze, preliminary questions about what had happened in the lesson, etc.) before asking "what do you think about X." Kim and Silver go on to argue that opportunities for meaningful reflection are inhibited when mentors act as feedback-providers but opened up when they act as facilitators of reflection.

The fifth section of the book examines current issues in research on CA and assessment to show how the evolving construct of oral proficiency can be measured formatively and summatively. Daşkin starts off this section by showing how a practice she identified as "Reference to a Past Learning Event" emerged as a formative assessment given by a teacher in a Turkish EFL classroom. In so doing, the teacher is able to check students' knowledge and thereby address any problems in understanding before continuing with the main instructional activity. Next, Huth explicates how an interactional learning target, in this case telephone openings, can be identified and formalized to inform teaching materials and tasks for classroombased assessment. However, Huth stresses that reconceptualizing learning targets in this way necessitates a reappraisal in other areas, particularly large-scale assessments like CEFR and ACTFL. Walters concludes this section by offering a well-balanced account of how CA and language testing mandates (e.g., "why that now" and "how that went," respectively) can come together to inform a classroom-based assessment. Using data from previous iterations of the test, Walters shows how their test design assesses students' ability to produce responses to compliments, assessments, and pre-sequences as well as responses to other-initiated repair moves. Although issues of validation persist, Walters provides a rigorous argument for how to create norms and frameworks from which inferences about learners' L2 oral performance can be warranted.

The last section of the book offers two discussion pieces that address two of the major recurring points in the book: (1) how to bridge the gap between researchers and practitioners; and (2) how to advance the field of L2 education in a way that is compatible with our current understanding of IC. First, Mori points out that the research presented in this book tends to fall into one of two categories: those that use $\mathrm{CA}$ for reflection on pedagogical practices and those that use CA to conceptualize objects of teaching and assessment. Grouping them in this way provides a useful lens through which to identify possible practitioner roles (e.g., teacher, teacher supervisor, curriculum or assessment developer). Additionally, Mori argues that bridging the gap involves not just "translating research," (i.e., one-way communication), but also listening to practitioners about their concerns. Pekarek Doehler ends the book by showcasing the progress that had been made thus far in the understanding and application of IC in real-world contexts (e.g., teacher supervision, assessment, and classroom interaction), while also noting areas in need of conceptual clarification and exciting new strands of research within IC. 
The major strengths of this book are its analytic rigor, breadth of research domains, and dedication to providing pedagogical implications. Each chapter, besides discussion pieces, carefully examines naturally occurring data with a combination of finely detailed transcripts that are often accompanied by screengrabs of relevant multimodal conduct. The researchers are thus able to provide a line-by-line analysis of what, exactly, is happening in various pedagogical contexts in order to either: (1) reveal pertinent practices that contribute to, or are indeed indicative of, language learning, or (2) show how CA can be used for pedagogical reflection. Furthermore, the breadth of research domains included in this book point in a promising direction for future research that represents various teaching contexts (e.g., ESL, EFL, CLIL), languages (e.g., Turkish, Swedish, Italian, etc.), and grade levels (e.g., primary, secondary, and post-secondary). Lastly, it was refreshing to see that, apart from the discussion pieces, each chapter ended with a section on pedagogical implications. As Mori notes, these sections varied from being fairly abstract (e.g., Huth or Majilesi) to listing an itinerary of concrete steps (e.g., Sert or Waring). The diversity of the kinds of pedagogical implications represented in these sections show how findings can be packaged in different ways depending on their intended audience (e.g., pedagogical implications based on teacher education frameworks are packaged in ways more suited to teacher supervisors). That being said, a synthesis of these pedagogical implications is mostly left to the reader, a task that may not be of interest unless the reader assumes the many roles of each author's intended audience (e.g., a teacher supervisor, a classroom teacher, a language program administrator, etc.).

Pulling together many threads of research on CA and pedagogical interaction, this book makes a crucial contribution to the field of applied linguistics by delivering a thoroughly rigorous account of how classroom interaction unfolds and contributes to second language acquisition, while clearly articulating how these findings can inform practice amongst language teaching professionals. This book will be of particular interest to those with a CA background, but it is sure to be an indispensable resource for anyone interested in understanding the complexity of classroom interaction.

\section{MARK ROMIG}

Teachers College, Columbia University 